\title{
Unsharp Masking, Countershading and Halos: Enhancements or Artifacts?
}

\author{
Matthew Trentacoste ${ }^{1}$ and Rafał Mantiuk ${ }^{2}$ and Wolfgang Heidrich ${ }^{1}$ and Florian Dufrot $^{2}$ \\ ${ }^{1}$ University of British Columbia, Canada \\ ${ }^{2}$ Bangor University, United Kingdom
}

\begin{abstract}
Countershading is a common technique for local image contrast manipulations, and is widely used both in automatic settings, such as image sharpening and tonemapping, as well as under artistic control, such as in paintings and interactive image processing software. Unfortunately, countershading is a double-edged sword: while correctly chosen parameters for a given viewing condition can significantly improve the image sharpness or trick the human visual system into perceiving a higher contrast than physically present in an image, wrong parameters, or different viewing conditions can result in objectionable halo artifacts. In this paper we investigate the perception of countershading in the context of a novel mask-based contrast enhancement algorithm and analyze the circumstances under which the resulting profiles turn from image enhancement to artifact for a range of parameters and viewing conditions. Our experimental results can be modeled as a function of the width of the countershading profile. We employ this empirical function in a range of applications such as image resizing, view dependent tone mapping, and countershading analysis in photographs and works of fine art.
\end{abstract}

\section{Introduction}

Local contrast enhancement is a powerful image processing technique, fundamental to many aspects of computer graphics such as image editing and tonemapping of HDR images. The countershading operation is one of the most common approaches to enhancing local contrast in images, where the local edge contrast is increased by adding gradients to either side of the edges. These gradients, known as countershading profiles, result from numerous algorithms. Regardless of the method of introduction, the result resembles that of the unsharp masking (UM) operator. Shown in Figure 1-red, unsharp masking increases local contrast by adding a high-pass image $\mathcal{H}_{\sigma}(Y)$ to the original image

$$
\mathcal{Y}=Y+\lambda \mathcal{H}_{\sigma}(Y)=(1+\lambda) Y-\lambda g_{\sigma} * Y
$$

where $\lambda$ is the contrast of the countershading and the highpass image $\mathcal{H}_{\sigma}(Y)$ is produced by subtracting a Gaussian blurred image $g_{\sigma} * Y$ from the original image $Y$.

Unsharp masking with a narrow high-pass filter can increase the apparent sharpness [Ney93] of the image, making fine details easier to identify. On the other hand, unsharp masking with a wide high-pass filter can increase the contrast of the regions adjacent to the edge [SKMS06], altering the overall impression of contrast in the image. Sufficiently wide unsharp masking profiles can even induce the Cornsweet illusion [KM88], where the entirety of adjacent regions change in appearance.

However, unsharp masking can also introduce objectionable countershading around an edge, frequently referred to as haloes. In these cases, the contrast enhancement detracts from the image, providing neither improved understanding nor aesthetic quality. The same operation can be interpreted as an enhancement in some cases, while it's interpreted as an artifact in others. We present a method of introducing perceptually-symmetric countershading profiles and conduct a perceptual study to determine the amount of local contrast that can be introduced by our operator without becoming objectionable. The goal is to find a boundary between where the result is considered contrast enhancement and artifact.

We discover the perception of countershading profiles follows a U-shaped characteristic, where certain width profiles are considered unacceptable even if only slightly visible, separating adjacent regions of both wider and narrower 


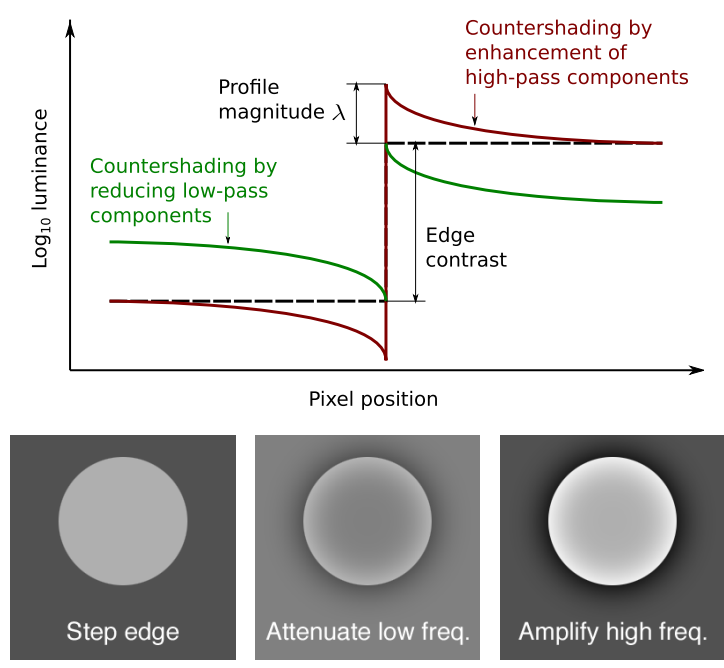

Figure 1: Countershading of a step edge (black dashed line) can be produced by either reducing low-frequency components (green line), or by amplifying the high-frequency components (red line). The former method is commonly used for tone-mapping while the latter for image enhancement.

countershading profiles with considerably higher levels of tolerated contrast. Figure 2 illustrates how the regions of indistinguishable and objectionable countershading vary with the width of the countershading profile and its amplitude. The important observation is that countershading profiles indistinguishable from a plain edge provide only very limited contrast enhancement; thus the region below objectionable countershading needs to be used to achieve high-quality results. We find that these regions correspond to the various semantic descriptions of the images resulting from different unsharp masking parameters. The trough of the curve consists of profiles considered haloes, while narrower profiles were perceived to sharpen image features and wider profiles were perceived to enhance contrast.

Our contributions in this paper include a model of acceptable countershading and several applications that employ it. Specifically:

- A model of the perceived appearance of countershading and an analysis of its relation to existing studies.

- A means of introducing countershading without amplifying fine details such as noise.

- Several applications to displaying content at different sizes including size-aware image resizing, control of tonemapping parameters and a viewer-adaptive display.

The benefit of this work is an understanding of how changes in the profile width affect the perceived quality of countershading, including those resulting from image downsampling and changes in viewing conditions such as display dimensions and distance. The results of our study can be used to avoid introducing artifacts when performing local contrast enhancement. However, the scope of our findings is

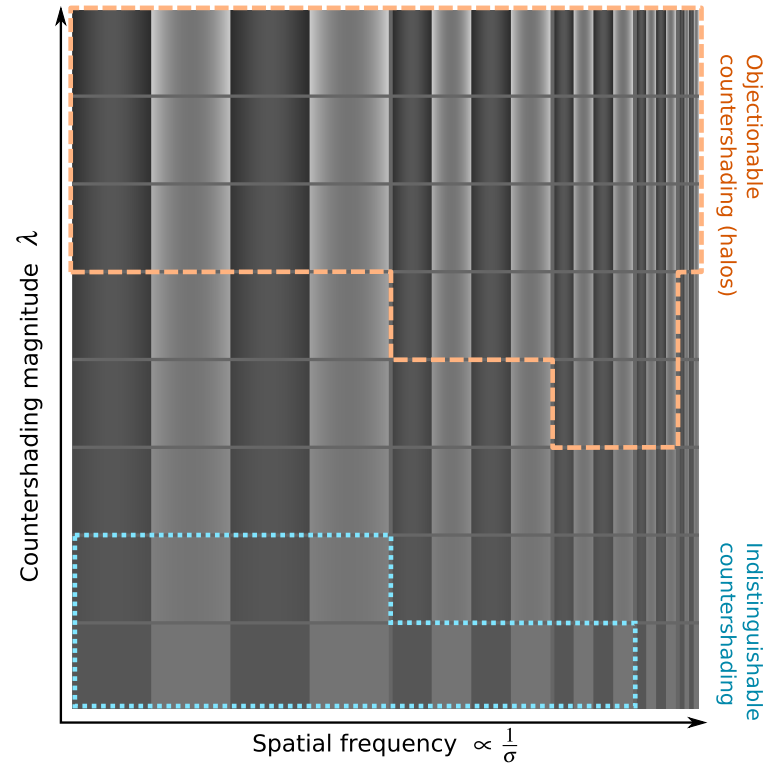

Figure 2: The square-wave pattern with a reduced amplitude of the fundamental frequency, resulting in countershading profiles. The regions of indistinguishable (from a step edge) and objectionable countershading are marked with dotted and dashed lines of different color. The higher magnitude of countershading produces higher contrast edges. But if it is too high, the result appears objectionable. The marked regions are approximate and for illustration and actual regions will depend on the angular resolution of the figure. Note that the spatial frequency shown on this plot is inversely proportional to the profile width $\sigma$, which is used in the rest of the paper.

limited by the small set of conditions and local contrast operators we were able to investigate in our study, a trade-off necessary to obtain accurate results for that set. Moreover, we show that our findings cannot be explained by the existing perceptual models; thus these models cannot be used to extrapolate the data to a broader range of conditions. As such, while relevant to numerous methods, our results are best taken as guidelines when processing complex images.

\section{Related Work}

Depending on the parameters used, the result of unsharp masking causes different changes in the appearance of the image. The choice of a small $\sigma$ in the high-pass filter increases the apparent sharpness [Ney93] of the image, which is the effect usually referred to as "unsharp masking" in literature [BA04]. However, narrow profiles do not significantly alter the perception of contrast [KM88]. The choice of a large $\sigma$ increases the contrast of the image, which is usually referred to as countershading. Most commonly, profile width and contrast, $\sigma$ and $\lambda$ respectively, are determined by the user and do not vary across the image. In this text, we 
refer to the technique as unsharp masking and the effect of local contrast enhancement it produces as countershading, regardless of the choice of $\sigma$.

Unsharp masking is known to excessively amplify the contrast of small features, especially noise, and a considerable body of work exists on how to choose optimal parameters to enhance contrast while avoiding unwanted amplification of noise. The most successful approaches have adapted the parameters to local image content including work by Polesel et al. [PRM00], Ramponi et al. [RSMY96] and Kim and Allebach [KA05] on adaptively determining $\lambda$ and work by Wang et al. [WWCX01] and Nowak and Baraniuk [NB98] on adaptively determining $\sigma$. However, most of this work attempts to address the issue of artifacts resulting from noise amplification, and not the artifacts caused by objectionable magnitude of countershading (haloes), which is the focus of our work.

Countershading is also frequently employed in local tonemapping operators, although not always intentionally. The operator by Chiu et al. [CHS*93] explicitly computed a high-pass image with a large $\sigma$ to reduce global contrast while retaining edge contrast. However, while reducing the dynamic range this naive approach introduced significant haloes. More recent operators like Durand and Dorsey [DD02] and Fattal et al. [FLW02] can also introduce countershading profiles, though they do not view them as a benefit. In general, countershading in local tonemapping is considered synonymous with halo artifacts or "gradient reversals". On the other hand, we view the particular combination of contrast and scale of the countershading to be responsible for the loss of image quality.

Countershading has been used in several other capacities to restore lost contrast and enhance scene understanding. Smith et al. have used countershading to restore color saturation lost during tonemapping [SKMS06] and while converting from color to greyscale [SLTM08]. Similarly, Luft et al. [LCD06] and Ritschel et al. [RSI*08] added countershading based on depth values to improve the recognition of objects in scenes.

Most related to our work is that of Krawczyk et al. [KMS07] on restoring luminance contrast lost during tonemapping. They propose an automated method that introduces countershading to an LDR tonemapped image to match the contrast of a reference HDR image. In an attempt to avoid introducing objectionable artifacts, they propose a perceptual model of just-detectable countershading. However, such a model relates to the detection of countershading profiles, not whether the countershading is considered objectionable, and is overly conservative. Additionally, Krawczyk's method is only applicable to the reproduction of contrast in an HDR image. We discuss their model in more detail in Section 5.2.

\section{Experiment Design}

In our perceptual experiment, we target the most general case of countershading operations: determining the magnitude at which a countershading profile of a given width becomes objectionable. While parameters of $\sigma$ and $\lambda$ responsible for the shape of the countershading profile smoothly vary over the space of possible values, a semantic shift from "enhancement" to "artifact" occurs along a boundary within that space. Our goal is to determine the boundary between the region of enhancements and the region of artifacts within the parameter space of the unsharp masking operator. Our study does not attempt to determine any of the other aspects of local contrast perception such as perceived contrast magnitude or the appearance desired by the user.

View setup. The images were presented on a 26" NEC LCD2690WUXi display with $1920 \times 1200$ resolution with a black level of $.45 \mathrm{~cd} / \mathrm{m}^{2}$ and a peak intensity of $213 \mathrm{~cd} / \mathrm{m}^{2}$. The experiment was run in a darkened room with no visible display glare. The viewing distance was $1 \mathrm{~m}$, resulting in a pixel Nyquist frequency of 30 cycles per visual degree.

Stimuli. The study consisted of six images, three test patterns of a single step edge of various contrasts, and three complex scenes, as shown in Figure 3. The process of adding countershading enhancement to an image is illustrated in Figure 4. Given a linear luminance image, the countershading is applied in the logarithmic domain to produce the profiles with the most symmetric appearance of lightness. To produce a high pass image for the enhancement, a Gaussianfiltered image is subtracted from an original. However, the enhancement is computed based on an edge-template, as opposed to using the original image. The template image contains the edges to be enhanced with smooth regions in

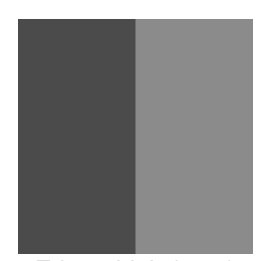

Edge - high (0.59)

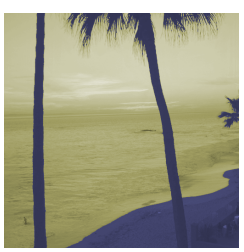

Palm beach (0.68)

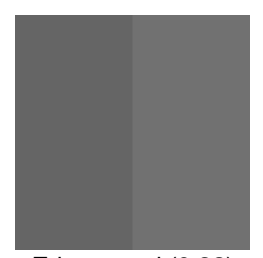

Edge - med (0.32)

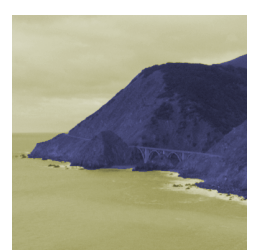

Coast (0.61)

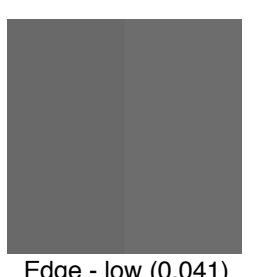

Edge - low (0.041)

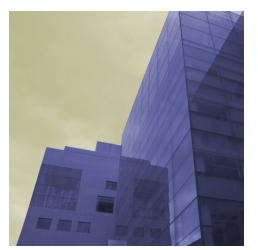

Building (0.39)
Figure 3: Images used in the perceptual experiment: 3 edges of different contrast and 3 images of complex scenes. The semi-transparent yellow-blue color mask is the edge template. The numbers in parenthesis denote the Michelson contrast of the edge. 


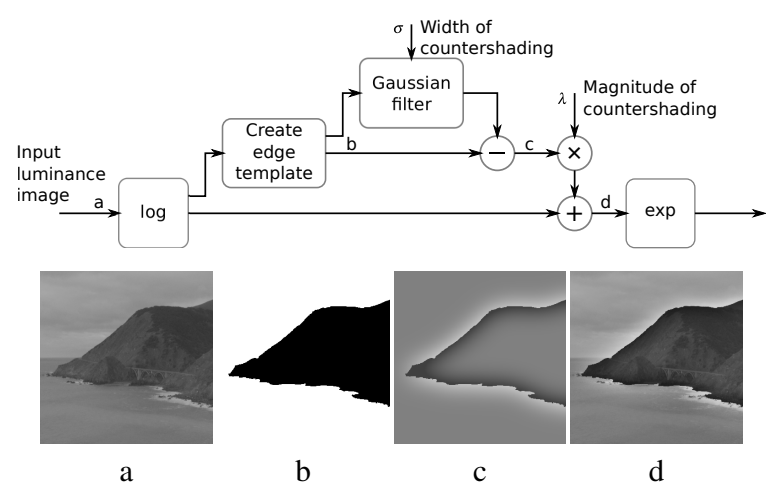

Figure 4: Generation of the countershading enhancement in an image. An edge template is used to precisely mark selected edges in the experiment, but is normally generated with an edge-preserving filter.

between, and can be produced by an edge-preserving filter such as that of Farbman et al. [FFLS08]. Using an edgetemplate ensures a constant increase in contrast along the edge and, more importantly, avoids the amplification of high frequency detail noted by Neycenssac [Ney93] which could distract subjects from evaluating the appearance of the countershading profiles. Ten countershading profile widths were used in the trials, were generated with high-pass filters with Gaussian $\sigma$ ranging from .009 to 4.6 visual degrees, equivalent to $.5-256 \mathrm{px}$ at $1 \mathrm{~m}$ viewing distance, increasing by factors of two.

Observers. 15 observers ( 9 male and 6 female) participated in the study. They were paid and unaware of the purpose of the experiment. The observer age varied from 23 to 37 with the average 29. All observers had normal or corrected-tonormal vision.

Procedure. After being presented an image with a countershading profile of width $\sigma$, the observers were asked to adjust the magnitude $\lambda$ of the countershading to the maximum level not considered an artifact. Both "artifact" and "objectionable" are subjective terms, and we relied on a noreference measure of the artifacts, where if the subject saw the image without knowing the original, they would say that it contained undesirable countershading. Each observer repeated the measurement for each of the 60 conditions $(6 \mathrm{im}$ ages $\times 10$ profile widths) twice, for a total of 120 trials each. In total, 1800 measurements were collected. The experiment was preceded with a training session to familiarize participants with the task. No data was recorded during the training phase, which was followed by three main sessions with voluntary breaks between them. The breaks were scheduled so that each session lasted less than 20 minutes.

Screening and outlier removal. Because of the subjective nature of the experiment, erroneous and inconsistent measurements are likely to be found in the data, which we fil- tered according to standard practices. First, we screened the participants using the procedure recommended for magnitude estimation experiments [IRB02, Sec. 2.3.1]. The procedure involves counting the number of trials in which the result of the observer lies outside the $\pm 2 \times$ standard deviation range and rejecting those observers for which a) more than $5 \%$ of the trials are outside that range; and b) the trials outside that range are evenly distributed so that the absolute difference between the counts of trials exceeding the lower and the upper bound of that range is not more than $30 \%$. Then, to further reduce the intra-observer variations, we eliminated those measurements for which the intra-observer standard deviation exceeded two times the mean standard deviation of all the measurements. The screening eliminated the data of two participants and the outlier removal removed $9 \%$ of the measurements.

\section{Experimental Results}

Figure 5 shows the results averaged over all observers. The most salient feature of all plots is the U-shaped characteristic, indicating a reduced tolerance to the halo effect for the medium profile widths, with the trough around 0.2 visual degrees. The black error bar in Figure 5a represents mean standard deviation across all data and thus illustrates variability in the measurements. The high variability comes from the subjective nature of the task; the objectionable threshold may have different meaning for each observer, resulting mostly in vertical shift of the plotted curves. However, the effect of the profile-width is clearly significant and observed across all the participants.

One of the most interesting observations is the difference in the characteristic between an isolated-edge and complex images, which is shown as the difference between the plots in Figures $5 \mathrm{a}$ and $5 \mathrm{~b}$. In the case of wide profiles, subjects tolerated higher contrast countershading in complex images than for isolated edges. This finding may be due to visual masking, present in complex images but absent in the edge stimuli. The difference between the two plots suggests that the measurements for simplified stimuli do not generalize to complex images.

The results for individual images are better aligned if the countershading profile is generated irrespective of the contrast of the underlying edge. The $\lambda$-values on the plots represent the magnitude of the countershading generated from an edge template (refer to Figure 4 ) with a fixed $\log _{10}$-contrast of 1 , rather than the contrast of an edge. While a small variation between narrow countershading profiles appears correlated with the underlying edge contrast, our data is not sufficient to model this effect. However, the effect disappears for large $\sigma$-values, which are the most relevant for an effective contrast enhancement. This observation suggests that profiles of the same magnitude should be used regardless of the contrast of the underlying edge.

For convenience, we fit a polynomial function with a lin- 


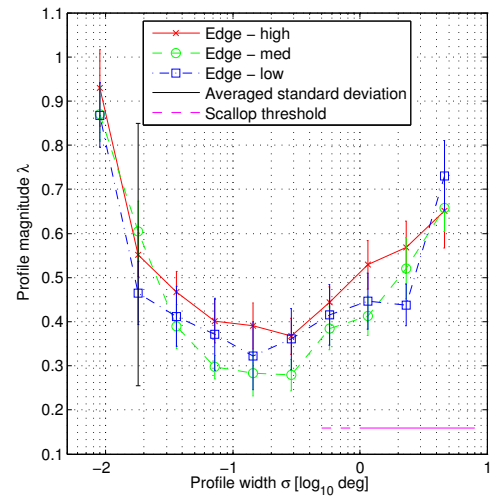

(a) Edges

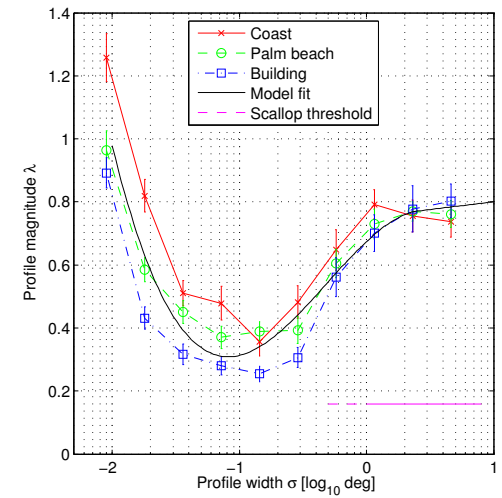

(b) Complex images

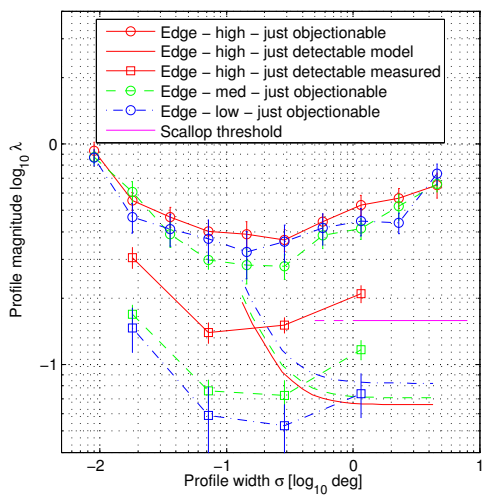

(c) Comparison with the detection thresholds

Figure 5: The results averaged over all participants, (a) for isolated edges, and (b) for complex images. The black line in (b) represents our model fit. (c) Our just-objectionable measurements (edges) compared to the just-distinguishable thresholds from Krawczyk et al. [KMS07] (lines with no markers) and our measurements (square markers). $\log _{10}$ scale was used for $\lambda$ in this plot for better visualization.

ear segment to the values averaged across all complex images. The $\lambda$-values for the just-objectionable countershading can be found from:

$$
\lambda= \begin{cases}-.249 \varsigma^{3}-.233 \varsigma^{2}+.377 \varsigma+.674 & \text { if } \varsigma \leq .418 \\ .048 \varsigma+.752 & \text { if } \varsigma>.418\end{cases}
$$

where $\varsigma=\log _{10}(\sigma)$. The model-fit is shown as a black continuous line in Figure $5 b$.

\section{Discussion}

\subsection{Relation to Detection}

The curve we obtained in the experiment appears to have a band-pass shape, similar to the one observed for the contrast sensitivity function (CSF) of the visual system. Therefore, it is interesting to see how the measured justobjectionable thresholds relate to the just-noticeable thresholds for countershading. Ihrke et al. [IRS*09] reported that the just-objectionable threshold is about 4 just-noticeabledifferences (JND) higher than the just-noticeable threshold. We examined this claim using a larger range of parameters and using stimuli with isolated profile widths.

We ran a study using the same procedure as described in Section 3 and the isolated edges from the previous experiment. Although 2-alternative-force-choice (2AFC) procedure seems to be the most appropriate for threshold measurements, it is not suitable for measuring the visibility of countershading profiles. This is because introducing countershading profiles either increases the contrast of the underlying edge, shown in red in Figure 1, or reduces the contrast between both sides of an edge, shown in green in the same figure. These contrast changes are much more detectable than the profile of the countershading and thus would be confused in a $2 \mathrm{AFC}$ procedure. For the same reason we were not able to predict the just-noticeable-thresholds for countershading profile using the visual difference predictor (VDP) [Dal93]. Such metrics detect the contrast change instead of the visibility of the profile itself.

The observers were asked to adjust the countershading strength, controlled by $\lambda$ parameter, until the gradient produced by countershading starts to appear. The results of the detection task are compared with the just-objectionable thresholds in Figure 5c. As expected, the JND thresholds are smaller and follow a similar shape as the just-objectionable thresholds. However, the differences between the contrast of the underlying edge are much more pronounced in the case of the JND thresholds.

To further investigate the relation between contrast and thresholds we conducted a second detection experiment, in which the $\log _{10}$-contrast of the underlying edge was varied across a wider range from 0.2 to 1.4 . We produced countershading by reducing low-pass components (green profile in Figure 1) instead of enhancing high-pass components. Our assumption was that the profiles produced by this method could be used with the VDP without confounding profile visibility with the edge-contrast changes. But later we discovered that the edge-contrast changes are too large for the VDP to correctly predict profile visibility.

The results of the second detection experiment, shown in Figure 6, further confirm that the detection of countershading depends on the edge-contrast. This is an argument against the hypothesis that the just-objectionable thresholds are related to the detection [IRS*09], as we did not find such strong dependence on edge contrast in the 


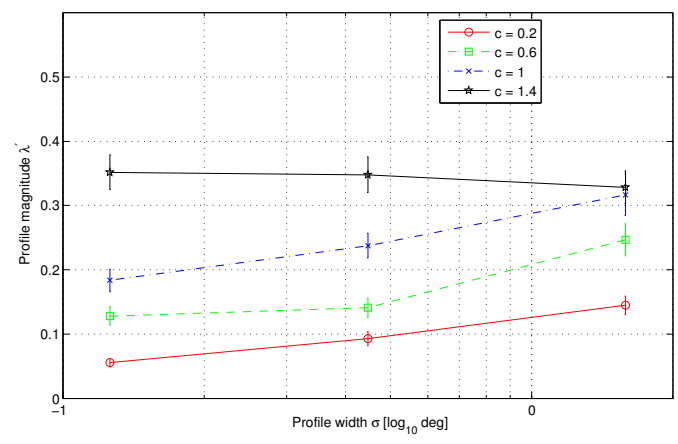

Figure 6: Measured thresholds for just-noticeable countershading profiles.

just-objectionable-threshold experiments. Another argument against such a relation is the effect of contrast constancy that 'flattens' the CSF characteristic for super-threshold contrast [GS75]. Since the just-objectionable countershading profiles consist mostly of super-threshold components, it is disputable whether their perception can be explained by the CSF.

\subsection{Relation to Other Studies}

The perceived effects of countershading and local contrast enhancement are well-studied. We review a selection of key work and discuss its relation to our own findings.

The studies on the Cornsweet illusion by Sullivan and Georgeson [SG77], Campbell et al. [CHR71, CHJ78] and Burr [Bur87] determined the contrast at which a countershaded edge becomes distinguishable from a step edge of equivalent contrast, the so called scalloping threshold. The horizontal magenta lines in Figures $5 a-5 c$ show the approximated values of scalloping threshold, after Kingdom and Moulden [KM88]. The thresholds apply only to larger $\sigma$-values because the countershading is always distinguishable from a square wave at spatial frequencies above $1 \mathrm{c} / \mathrm{deg}$ [SG77], which approximately corresponds to $\sigma<1$ for our Gaussian-based profiles. Very low scalloping thresholds clearly show that the countershading is likely to be noticeable for most practical cases; and thus the justobjectionable threshold is more relevant for contrast enhancement applications. For a more complete discussion of scalloping, see Kingdom and Moulden [KM88] for a comprehensive review.

Lin et al. [LGK06] studied the perceptual impact of edge sharpness. In their study, they processed images using an unsharp masking filter with a fixed $\sigma=1.4 \mathrm{px}$ at edge locations only to avoid noise amplification. This width equates to .04 spatial degrees in their viewing setup, roughly corresponding to the third of our tested widths. The filter was applied to all edges in the image, and the amount of countershading introduced was proportional to the underlying edge contrast. Subjects ranked the perceived quality of images for contrasts of different magnitudes and Lin et al. computed the most desirable and highest tolerated contrast.

Based on Dooley's and Greenfield's [DG77] theoretical model of countershading perception, Krawczyk et al. [KMS07] proposed a visual model of just-detectable countershading, which was used to adaptively introduce countershading in images. The main assumption behind their algorithm is that the countershading becomes objectionable as soon as the profiles become visible. The comparison of our data with the scalloping thresholds in Figures 5a and $5 \mathrm{~b}$ demonstrates that it is not the case. We reproduced their model and computed its predictions for the isolated edges from our experiment, assuming the threshold-versusintensity (t.v.i.) value equal to $5 \%$ and no masking. The model predictions, plotted as continuous lines in Figure 5c, approximate our detection experiment results (square markers) for small edge contrast and large sigmas, but show little correlation with our data for other conditions.

Ihrke et al. [IRS*09] performed a perceptual evaluation of the work on 3D unsharp masking by Ritschel et al. [RSI*08]. The authors tested the preferred value of their magnitude parameter $\lambda$ for profiles of several different widths, measured on object surfaces, opposed to image-space. Interestingly, they did not find their results varied with the width of the countershading profiles. The depth-dependency of their algorithm means that profiles of varying widths were added to their scenes, making it hard to ascertain the magnitude level associated with a single countershading width. Additionally, they only tested images of complex scenes with a narrow set of profiles.

\section{Applications}

We have implemented a number of simple applications that relate to objectionable countershading, including improved unsharp masking, resize-aware contrast enhancement and tone mapping, and countershading profile analysis. The purpose of these examples is to demonstrate the breadth of topics for which our study is relevant, and any of these tools could be made more sophisticated.

As noted in Section 5, the perception of countershading profiles strongly depends on the spatial frequency at which they appear. All the images assume viewing of this paper as printed on letter-size paper $\left(8.5^{\prime \prime} \times 11^{\prime \prime} / 11.6 \times 27.9 \mathrm{~cm}\right)$ page viewed from a distance of $18 "(45.7 \mathrm{~cm})$. Additionally, some of the effects are subtle and we suggest looking at the electronic copy of the paper.

\subsection{Artifact-free unsharp masking}

Neycenssac [Ney93] noted that unsharp masking introduces contrast through two separate mechanisms: the addition of countershading profiles around edges and the amplification of existing image features. Convolution with a Gaussian of 
width $\sigma$ removes all features smaller than $\sqrt{\sigma}$ [Lin94], so those features, including noise, will be present high-pass image $\mathcal{H}_{\sigma}(Y)$ at their original contrast. To achieve effective countershading, without undesired detail amplification, it is necessary to suppress unwanted details in the high-pass image.

Conventional approaches adaptively scale unsharp masking parameters $\lambda$ and $\sigma$ to avoid introducing unwanted features in $\mathcal{H}_{\sigma}(Y)$ from being introduced into the final image. Conversely, we generate an alternative high-pass image that does not contain the undesirable features in the first place. We replace the conventional high-pass image $\mathcal{H}_{\sigma}(Y)$, based on the difference between the image and a Gaussian-blurred copy $g_{\sigma}$

$$
\mathcal{H}_{\sigma}(Y)=Y-g_{\sigma} * Y
$$

with a modified version $\mathcal{H}_{\sigma}^{\prime}(Y)$ based on a template function $T_{\sigma}$ that removes features smaller than the $\sqrt{\sigma}$ from the image while retaining high-frequency edges:

$$
\mathcal{H}_{\sigma}^{\prime}(Y)=T_{\sigma}(Y)-g_{\sigma} * T_{\sigma}(Y)
$$

Edge-preserving smoothing filters, especially the weighted least squares (WLS) framework of Farbman et al. [FFLS08], provide a very good approximation of $T_{\sigma}$. To do so, the frequency response of the WLS filter must be calibrated to match that of $g_{\sigma}$ used by $\mathcal{H}$. The frequency response of a Gaussian filter of width $\sigma$ is another Gaussian of width $1 / \sigma, \mathscr{G}_{\sigma}(\omega)=e^{-\omega^{2} /\left(2 / \sigma^{2}\right)}$. The frequency response of the WLS filter for a region without significant edges is

$$
\mathscr{F}_{\gamma, \alpha}(\omega)=\frac{1}{1+\gamma \omega^{2}\left(|\partial \ell|^{\alpha}+\varepsilon\right)^{-1}},
$$

where $\gamma$ controls the spatial extent of the function, $\alpha$ is the edge-stopping parameter, $\partial \ell$ is the average partial difference of the log-luminance of the image and $\varepsilon$ avoids division by zero. We choose Farbman's value of $\alpha=1.2$ and an average pixel difference of .04 to approximate the gradient magnitude of flat regions of images and solve the $\gamma$ that minimizes the least-squares difference between $\mathscr{G}_{\sigma}$ and $\mathscr{F} \gamma$. For $\sigma=1$, the equivalent value of $\gamma_{1}=0.027$, given our choice of parameters, and values equivalent to other $\sigma$ are determined by $\gamma_{\sigma}=\sigma^{2} \gamma_{1}$

The corresponding WLS filter $F_{\lambda}$ removes features smaller than $\sqrt{\sigma}$ while preserving edges. Figure 7 compares conventional unsharp masking to the template image approach, which successfully removes small details from the high-pass image. We use this technique to demonstrate the ability of our model from Section 4 and introduce acceptable countershading to a number of images included in the supplementary material. WLS filtering does not smooth across

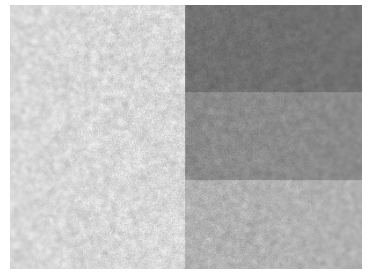

a: original $Y$

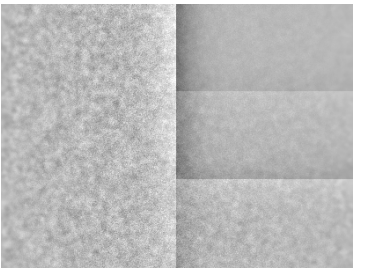

c: naïve high-pass $\mathcal{H}_{\sigma}(Y)$

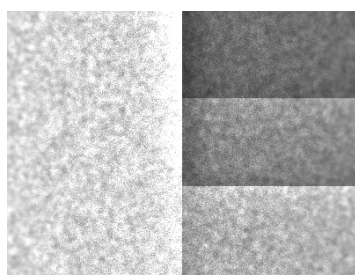

e: naïve countershade

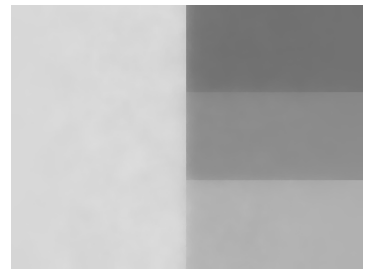

b: template $T_{\sigma}(Y)$

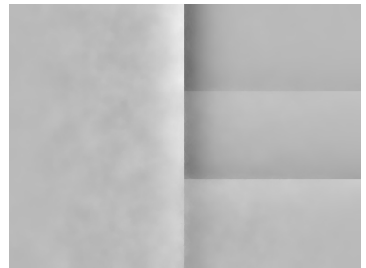

d: template high-pass $\mathcal{H}_{\sigma}^{\prime}(Y)$

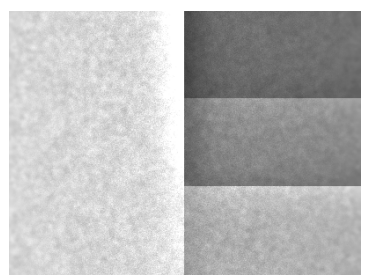

f: template countershade
Figure 7: Comparison of the original image $Y$ with the result of the template function $T_{\sigma}(Y)$ for the generated high-pass image and countershaded result. The naïve high-pass image retains the noise of the original and amplifies it in the result, while the template version does not. In this case the countershading magnitude has been chosen to make the difference between operators easily visible.

edges and tone manipulation predominantly occurs in regions without sharp edges. Conversely, our operator predominantly adjusts the contrast of regions around sharp edges.

\subsection{Resize-aware countershading}

Conventionally, countershading is applied relative to the full-size image, and the resulting contrast manipulation is not altered in response to different displayed sizes, whether due to resampling or different display dimensions. Resizing an image with acceptable countershaded edges can cause the profiles around those edges to move into the objectionable region, as shown in Figure 8.

Figure 8 a shows an edge with $1.84^{\circ}$ countershading profiles near the just-acceptable contrast. Shrinking that image by a factor of $10 \times$ causes those profiles to move into the objectionable region and appear as haloes. In order to regain acceptable profiles, the current combination of profile contrast and width applied before resizing must be projected back outside the objectionable region. Fig $8 \mathrm{~b}$ and $8 \mathrm{c}$ show the result of projecting along only the contrast axis or only 

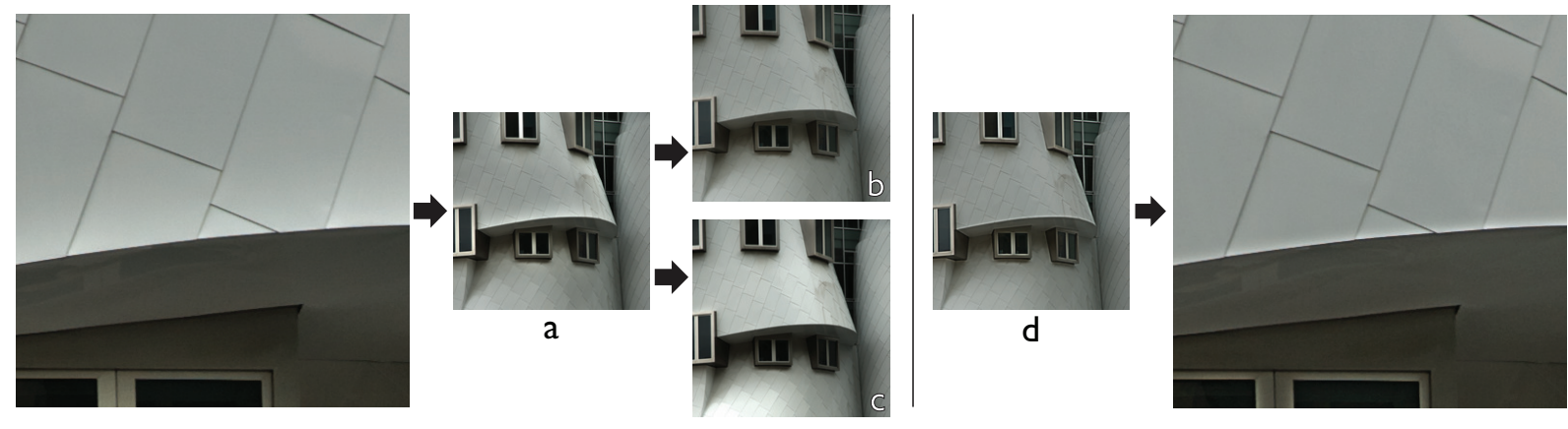

Figure 8: Downsizing an image (a) transforms a $1.84^{\circ}$ countershading profile to a smaller one, where the threshold of objectionable magnitude is significantly reduced, causing objectionable haloes. These artifacts can be remedied by adjusting the magnitude of the countershading (b) to correspond to the new angular size of the profiles or adjusting the width of the countershading (c) to compensate for the downsample factor, or some combination thereof. Neither image in this simple case appears exactly the same as the full-size, but they do not include objectionable artifacts. The same applies in the opposite case, when a sharpened image is enlarged $(d)$.

the width axis, respectively. The resulting appearance depends on the projection, where primarily adjusting contrast reduces the overall image contrast enhancement, while primarily adjusting width approximates a global contrast adjustment. Algorithms can choose how to steer that projection to yield one of those two outcomes, or an intermediate image appearance.

The same issue also must be considered when enlarging images. Contrasts acceptable for very narrow profiles will become objectionable as they are widened, as shown in Figure $8 \mathrm{~d}$. While less frequent, this scenario can occur if a user is adjusting the contrast of a sharpening filter in a preview of less than $100 \%$ of the full image size.

\subsection{Local tonemapping operators}

The same consideration to the displayed scale of countershading can be applied to other operations. Local tonemapping is frequently associated with introducing objectionable haloes. In some cases, the perception of poor algorithm performance may be due be a mismatch between the scale for which the image was intended and the scale at which it is being displayed.

We use Durand and Dorsey's [DD02] Office image, which is $2000 \times 1312 \mathrm{px}$ in size. Their algorithm calls for the $\sigma_{s}$ of the bilateral filter to be $2 \%$ of the image size, in this case 26 px. Viewing the image $1-$ to- 1 pixels on a $26^{\prime \prime} 1920 \times 1200$ display from a distance of $2 ", \sigma_{s}$ is equivalent to $0.70^{\circ}$. We observe the artifacts to be acceptably low under these conditions. However, presenting that image the width of a single column of this paper at our 18 " viewing distance, the same $\sigma_{s}$ is equivalent to $0.28^{\circ}$, a spatial frequency at which we are considerably more sensitive to haloes.

After fixing the bilateral $\sigma_{s}$ to be equivalent to $0.70^{\circ}$
(66 px in the case of the full-resolution image displayed the width of one column) the haloes are below the objectionable threshold for the image in this paper, as can be seen in Figure 9. We have not entirely removed the haloes from the image, as can be seen when viewing the image from a larger distance. We have simply ensured the profile contrasts are below the objectionable threshold for the given viewing conditions.

\subsection{Viewer-adaptive display}

The underlying theme of these applications is that the perception of countershading strongly depends on the width of the profile from the point of the viewer. Algorithms can easily account for the dimensions and resolution of the display, but fail to account for the fact that the perceived profile width also depends on the distance between observer and display. We created a setup with a viewer-adaptive display, which determines the distance of a viewer from the screen using head-tracking, and then adjusts countershading profiles accordingly (details in the video). The goal is to maximize contrast enhancement while avoiding haloes to appear, especially at larger viewing distances (refer to Figure 5). We can achieve the adjustment in two different ways: either by keeping the width of the profiles constant on the screen and adjusting the magnitude of the distortion, or by changing the width of the profiles on the screen so that their angular size from the viewer point stays the same. We found the first approach to be less disruptive to image content while the second to provide stronger contrast enhancement.

\subsection{Countershading analysis}

Many images already contain countershading, including some works of fine art such as Seurat's Le Bec du Hoc, shown in Figure 10. In fact, countershading originated in the 

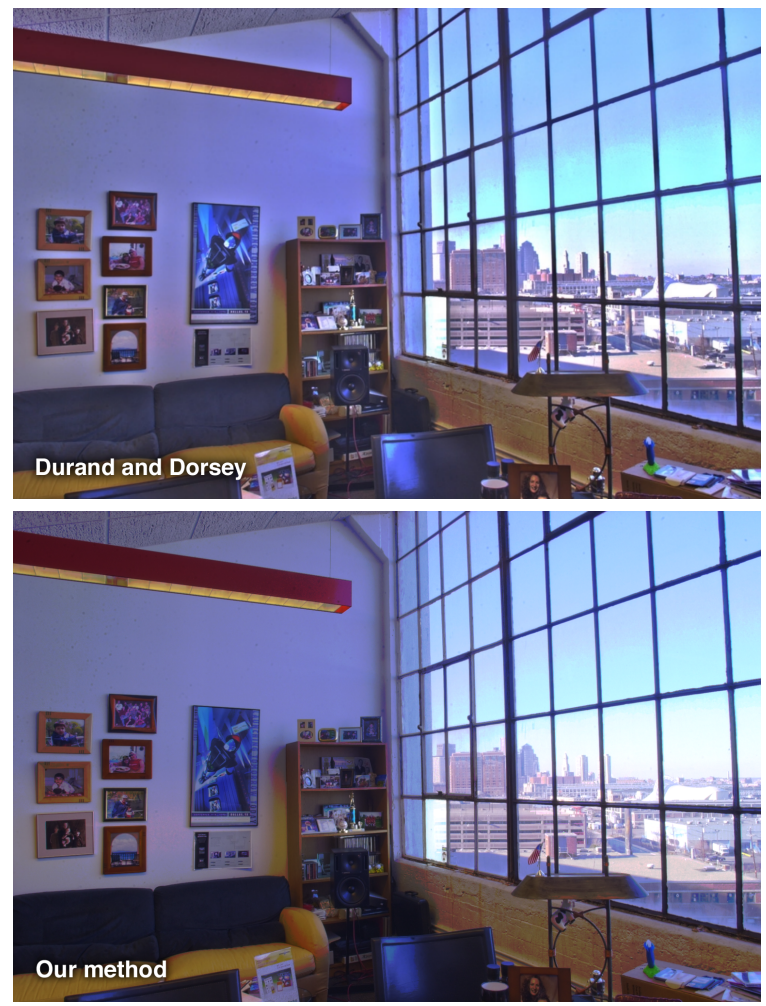

Figure 9: A comparison between the $\sigma_{s}$ specified in Durand and Dorsey [DD02] (top) and our $\sigma_{s}$ chosen for the size of the image in this paper (bottom). The contrast of the countershading has not changed, the profiles have been sufficiently widened so the contrast is no longer objectionable.

fine arts, where artists gave images the appearance of higher contrast than the medium could otherwise convey. To date, the majority of countershading operation have focused on introducing profiles to images. Few, if any, of the techniques that utilize countershading provide insight into images already containing countershading profiles.

To analyze the countershading present in an image we use an extreme case of image abstraction followed by an analysis-by-synthesis estimation of the countershading profiles. We observe that if a single iteration of an edgepreserving filter removes texture details, multiple iterations remove any low-amplitude intensity changes, including the countershading profiles. We employ the iterative version of the WLS framework [FFLS08] to obtain a texture-free layer $D_{s}$, and then repeat several more iterations to obtain a countershading-free layer $D_{t}$. The layer $D_{t}$ consists of nearly uniform regions of color separated by sharp edges like the template images described earlier. We then segment the template image and solve for the Gaussian centered at each edge position that best approximates the countershading present: $D_{t}-D_{s}$. Estimation is performed independently for each side of the edge to account for asymmetries in the profiles.
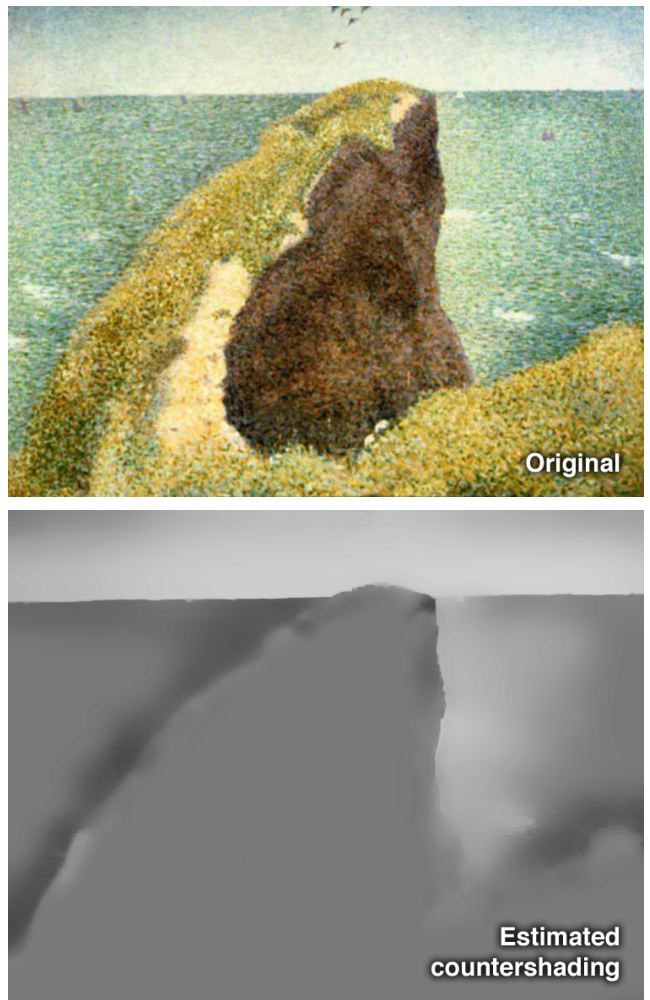

Figure 10: We performed a multi-scale decomposition of Seurat's Le Bec du Hoc (top) and estimated the countershading profiles on each side of the edges separated the land, sea, and sky portions (bottom).

The result is an approximation of profile width and magnitude at each edge location, shown in Figure 10 (bottom). Further investigation with this approach may afford a deeper insight into how countershading has been used artistically.

\section{Conclusion}

In this study we measured conditions under which countershading profiles are perceived as objectionable. We found a strong effect of the width and magnitude of the profile and a much weaker effect of the underlying image content. Unlike previous studies on the detection of countershading profiles [SG77, KMS07] or their matching contrast [DG77], our work focuses on the aesthetics of countershading. We have shown several applications where our model, combined with edge-preserving smoothing, can be used to improve upon existing countershading approaches, as well as enable some new possibilities. Improvements to existing methods include a new unsharp masking operator that avoids noise amplification, more accurate resizing of countershading profiles and halo-free local tonemapping. We also present a means of estimating countershading in existing images, including fine art.

We expected to relate our results to existing psychophys- 
ical studies to produce a comprehensive model of the perception of countershading aesthetics. Instead, we discovered an effect that does not seem to conform to existing perceptual models. Given this finding, the results of our study are best used as a heuristic until a more comprehensive study, including contrast masking and larger variation of the underlying edge contrast across a wider range of images, can be conducted.

To conclude, the perceived quality of countershading is strongly affected by changes in profile width, including those resulting from image downsampling and changes in viewing conditions such as display dimensions and distance. Any change to the size of the image, from the observer's point of view, affects countershading profile widths and can convert acceptable profiles into objectionable ones, implying this observation is very relevant to a large group of image enhancement algorithms. It is our suspicion that a number of algorithms said to introduce haloes may in fact produce acceptable results, but suffer from the disparity in size between authors' monitors and their printed reproductions.

\section{Acknowledgements}

This work was partly supported by the EPSRC research grant EP/I006575/1.

\section{References}

[BA04] Badamchizadeh M. A., Aghagolzadeh A.: Comparative study of unsharp masking methods for image enhancement. In Proc. Intl. Conf. Image \& Graph. (2004), pp. 27-30. 2

[Bur87] BURR D.: Implications of the Craik-O'Brien illusion for brightness perception. Vis. Res. 27, 11 (1987), 1903-1913. 6

[CHJ78] Campbell F. W., Howell E. R., Johnstone J. R.: A comparison of threshold and suprathreshold appearance of gratings with components in the low and high spatial frequency range. J. Physiol. (Lond.) 284 (1978), 193-201. 6

[CHR71] CAmpbell F. W., Howell E. R., Robson J. G.: The appearance of gratings with and without the fundamental Fourier component. J. Physiol. (Lond.) 217 (1971), 17P-18P. 6

[CHS*93] Chiu K., Herf M., Shirley P., Swamy S., Wang C., Zimmerman K.: Spatially nonuniform scaling functions for high contrast images. In Proc. Graphics Interface (1993), pp. 245-253. 3

[Da193] DALY S.: The visible differences predictor: an algorithm for the assessment of image fidelity. Digital images and human vision (1993), 179-206. 5

[DD02] DuRAND F., DorSEY J.: Fast bilateral filtering for the display of high-dynamic-range images. ACM Trans. Graph. 21 (2002), 257-266. 3, 8, 9

[DG77] Dooley R., Greenfield M.: Measurements of edgeinduced visual contrast and a spatial-frequency interaction of the Cornsweet illusion. J. Opt. Soc. Am. 67, 6 (1977), 761-765. 6, 9

[FFLS08] Farbman Z., FatTal R., Lischinski D., SZELISKI R.: Edge-preserving decompositions for multi-scale tone and detail manipulation. ACM Trans. Graph. 27 (2008), 67:1-67:10. 4, 7,9
[FLW02] FatTAl R., Lischinski D., WeRman M.: Gradient domain high dynamic range compression. ACM Trans. Graph. 21, 3 (2002), 249-256. 3

[GS75] Georgeson M. A., Sullivan G. D.: Contrast constancy: deblurring in human vision by spatial frequency channels. J. Physiol. 252, 3 (1975), 627-656. 6

[IRB02] ITU-R-BT.500-11: Methodology for the subjective assessment of the quality of television pictures, 2002. 4

[IRS*09] Ihrke M., Ritschel T., Smith K., Grosch T., MYSZKowsKi K., SEIDEL H.-P.: A perceptual evaluation of 3D unsharp masking. Human Vision and Electronic Imaging (2009), 12. 5,6

[KA05] Kim S. H., AllebaCh J. P.: Optimal unsharp mask for image sharpening and noise removal. J. of Electronic Imaging 14, 2 (2005), 023005. 3

[KM88] Kingdom F., Moulden B.: Border effects on brightness: A review of findings, models, issues. Spatial Vision 3, 4 (1988), 225-262. 1, 2, 6

[KMS07] KrawcZyk G., Myszkowski K., Seidel H. P.: Contrast restoration by adaptive countershading. In Eurographics (2007). 3, 5, 6, 9

[LCD06] LufT T., Colditz C., Deussen O.: Image enhancement by unsharp masking the depth buffer. ACM Trans. Graph. 25,3 (2006), 1206-1213. 3

[LGK06] Lin W., Gai Y., Kassim A.: Perceptual impact of edge sharpness in images. Vision, Image and Signal Processing, IEE Proceedings - 153, 2 (2006), 215 - 223. 6

[Lin94] LINDEBERG T.: Scale-space theory: A basic tool for analyzing structures at different scales. J. Applied Statistics 21, 1 (1994), 225-270. 7

[NB98] NOWAK R. D., BARANiUK R. G.: Adaptive weighted highpass filters using multiscale analysis. IEEE Trans. Image Process. 7 (1998), 1068-1074. 3

[Ney93] Neycenssac F.: Contrast enhancement using the laplacian-of-a-gaussian filter. CVGIP: Graphical Models and Image Processing 55, 6 (1993), 447 - 463. 1, 2, 4, 6

[PRM00] Polesel A., RAMPONI G., Mathews V.: Image enhancement via adaptive unsharp masking. IEEE Trans. Image Proc. 9, 3 (2000), $505-510.3$

[RSI*08] Ritschel T., SMith K., Ihrke M., Grosch T., MYSZKOWSKI K., SEIDEL H.-P.: 3D unsharp masking for scene coherent enhancement. ACM Trans. Graph. 27, 3 (2008). 3, 6

[RSMY96] Ramponi G., Strobel N. K., Mitra S. K., Yu T.-H.: Nonlinear unsharp masking methods for image contrast enhancement. Journal of Electronic Imaging 5, 3 (1996), 353366. 3

[SG77] Sullivan G., GeORGeSon M.: The missing fundamental illusion: Variation of spatio-temporal characteristics with dark adaptation. Vis. Res. 17, 8 (1977), 977-981. 6, 9

[SKMS06] Smith K., Krawczyk G., Myszkowski K., SeIDEL H.: Beyond tone mapping: Enhanced depiction of tone mapped HDR images. Eurographics 25, 3 (2006), 427-438. 1, 3

[SLTM08] SMith K., LANDES P., Thollot J., MyszKowsKi K.: Apparent greyscale: A simple and fast conversion to perceptually accurate images and video. Eurographics (2008). 3

[WWCX01] Wang Y.-P., Wu Q., Castleman K. R., Xiong $\mathrm{Z}$.: Image enhancement using multiscale differential operators. In IEEE Proc. of the Acoustics, Speech, and Signal Processing (2001), pp. 1853-1856. 3 